\title{
THE SEMIGROUP OF COMBINATORIAL CONFIGURATIONS
}

\author{
MARIA BRAS-AMORÓS, KLARA STOKES
}

\begin{abstract}
We elaborate on the existence and construction of the so-called combinatorial configurations. The main result is that for fixed degrees the existence of such configurations is given by a numerical semigroup. The proof is constructive giving a method to obtain combinatorial configurations with parameters large enough.
\end{abstract}

\section{INTRODUCTION}

In the literature a combinatorial configuration [6] (or a partial linear space [1]) is defined as a particular case of a so-called incidence structure. Here, for simplicity, we chose to define it as a particular case of bipartite graph. Since incidence structures and bipartite graphs are essentially the same, our choice to use bipartite graphs does not introduce any ambiguity. We define a $(v, b, r, k)$-combinatorial configuration as a connected bipartite graph with $v$ vertices on one side, each of them of degree $r$, and $b$ vertices on the other side, each of them of degree $k$, and with no cycle of length 4 .

There are many results on the existence of combinatorial configurations. For instance in Gropp's papers [3, 4, 5, 6] and in Grünbaum's book [7. Gropp states in his references that the next two conditions are necessary for the existence of a $(v, b, r, k)$-configuration. $\mathrm{P} 1: v r=b k ; \mathrm{P} 2: v \geq r(k-1)+1$. In particular, for $k=3$ he proves that $\mathrm{P} 1$ and $\mathrm{P} 2$ are also sufficient. The next theorem by Gropp guarantees the existence of large configurations and, in fact, the existence of any configuration satisfying the necessary conditions with sufficiently large $v$ (and so $b)$. Its limitation is the restriction on the choice of the parameters $r, k$.

Theorem 1. [4, Theorem 3.13] For given $k$ and $r$ with $r=t k$ there is a $v_{0}$ depending on $k, t$ such that there is a $(v, b, r, k)$-configuration for all $v \geq v_{0}$ satisfying $P 1$ and $P 2$.

In this short note we will generalize this result by showing that for any fixed $k \geq 2$ and for any fixed $r \geq 2$, the set of tuples $(v, b)$ for which a $(v, b, r, k)$-combinatorial configuration exists is in bijection with a numerical semigroup.

\section{The submonoid of $(r, k)$-COnfigurable tuples}

We say that the tuple $(v, b, r, k)$ is configurable if a $(v, b, r, k)$ configuration exists. It is immediate to prove that if $(v, b, r, k)$ is configurable then $v r=b k$ and consequently there exists $d$ such that $v=d \frac{k}{\operatorname{gcd}(r, k)}$ and $b=d \frac{r}{\operatorname{gcd}(r, k)}$. So, to each

This work was partly supported by the Spanish Government through projects TIN2009-11689 "RIPUP" and CONSOLIDER INGENIO 2010 CSD2007-00004 "ARES", and by the Government of Catalonia under grant 2009 SGR 1135. 
configurable tuple $(v, b, r, k)$ we can assign an integer $d$ and two different configurable tuples $(v, b, r, k)$ will have different integers $d$. Let us call $D_{r, k}$ the set of all possible integers $d$ corresponding to configurable tuples $(v, b, r, k)$. That is,

$$
D_{r, k}=\left\{d \in \mathbb{N}_{0}:\left(d \frac{k}{\operatorname{gcd}(r, k)}, d \frac{r}{\operatorname{gcd}(r, k)}, r, k\right) \text { is configurable }\right\} .
$$

Our aim is to study $D_{r, k}$. We will consider the empty graph to be also a configuration and consequently $0 \in D_{r, k}$ for all pair $r, k$. Obviously $D_{r, k}=D_{k, r}$ and $D_{1, k}=\{0, k\}$. We will prove that if $r, k>1$ then $D_{r, k}$ is a numerical semigroup, that is, a subset of $\mathbb{N}_{0}$ containing 0 , closed under addition and with a finite complement in $\mathbb{N}_{0}$. A general reference on numerical semigroups is [8]. If $a_{1}, \ldots, a_{l}$ are coprime then the set $\left\{n_{1} a_{1}+\cdots+n_{l} a_{l}: n_{1}, \ldots, n_{l} \in \mathbb{N}_{0}\right\}$ is a numerical semigroup and it is called the semigroup generated by $a_{1}, \ldots, a_{n}$ and denoted by $\left\langle a_{1}, \ldots, a_{l}\right\rangle$.

In the next section we will give a complete description of $D_{2, k}$ and in the last one we will study the case $r \geq 3$.

\section{THE CASE $r=2$}

There is a natural bijection between $(v, b, 2, k)$-configurations and $k$-regular connected graphs with $b$ vertices and $v$ edges. Two vertices in the graph share an edge if and only if the corresponding nodes in the configuration share a neighbor and viceversa. The next well-known lemma is the key result for describing $D_{2, k}$.

Lemma 1. (1) If $k$ is even, a connected $k$-regular graph with $b$ vertices exists if and only if $b \geq k+1$.

(2) If $k$ is odd, a connected $k$-regular graph with $b$ vertices exists if and only if $b$ is even and $b \geq k+1$.

Proof. (1) By definition, any $k$-regular graph must have a number of vertices at least $k+1$. Conversely, suppose $b \geq k+1$. Consider a set of vertices $x_{1}, \ldots, x_{b}$. Put an edge between $x_{i}$ and $x_{j}$, with $i \leq j$, if $j-i \leq k / 2$ or $i+b-j \leq k / 2$. This gives a connected $k$-regular graph with $b$ vertices.

(2) By definition, any $k$-regular graph must have a number of vertices at least $k+1$. Now, since the number of edges is $k b / 2$ this means that $k b$ must be even and since $k$ is odd $b$ must be even. Conversely, suppose $b$ is even and $b \geq k+1$. Consider a set of vertices $x_{1}, \ldots, x_{b}$. Put an edge between $x_{i}$ and $x_{j}$, with $i \leq j$, if $j-i \leq(k-1) / 2$ or $i+b-j \leq(k-1) / 2$. Put also edges between $x_{i}$ and $x_{i+b / 2}$ for $i$ from 1 to $b / 2$. This gives a connected $k$-regular graph with $b$ vertices.

Corollary 1. $D_{2, k}= \begin{cases}\langle k+1, k+2, \ldots, 2 k+1\rangle & \text { if } k \text { is even, } \\ \left\langle\frac{k+1}{2}, \frac{k+1}{2}+1, \frac{k+1}{2}+2, \ldots, k\right\rangle & \text { if } k \text { is odd. }\end{cases}$

4. THe CASE $r \geq 3, k \geq 3$

4.1. The set $D_{r, k}$ is non-trivial.

Lemma 2 (Sachs [9]). For any integer $n \geq 3$ and any $\gamma \geq 2$ there exists an $n$-regular graph with girth at least $\gamma$.

Lemma 3. For any pair of integers $r, k$, there exists at least one non-zero integer in $D_{r, k}$ for all $r, k$. 
Proof. The cases in which $r \leq 2$ or $k \leq 2$ have been proved in the previous sections. So, we can assume that $r \geq 3$ and $k \geq 3$. Consider the complete bipartite graph $K_{r, k}$. From basic graph theory we know that we can take a subset of $r+k-1$ edges in $K_{r, k}$ such that they connect all $r+k$ vertices and no cycle is formed (i.e., a generating tree). Let $A$ be the set of the $r k-r-k+1$ remaining edges of $K_{r, k}$.

Let $n=r k-r-k+1$ be the number of edges in $A$. Notice that since $r$ and $k$ are at least 3 then $n \geq 3$. Consider an $n$-regular graph $G$ with girth at least 5 as in Lemma 2 and consider as many copies of $K_{r, k}$ as vertices in $G$. Associate each copy of $K_{r, k}$ to a different vertex in $G$. For each edge $e$ in $G$, take the copies of the graphs $K_{r, k}$ corresponding to the ends of $e$ and swap one edge $x y$ in $A$ in the first copy and one edge $x^{\prime} y^{\prime}$ in $A$ in the second copy for $x y^{\prime}$ and $x^{\prime} y$ (here we abused notation using the same letter $A$ for different copies of it). This can be done in a way such that every time we take one edge in $A$ corresponding to a given copy of $K_{r, k}$, the edge is different.

It is easy to check that we obtain a non-trivial $(r, k)$-biregular bipartite graph with girth at least 5 .

\subsection{The set $D_{r, k}$ is a numerical semigroup.}

Lemma 4. Suppose we have a $(v, b, r, k)$-configuration with $r, k \geq 2$. There exist three edges in the configuration such that the six ends are all different.

Proof. Since no cycle of length 4 exists and $r, k \geq 2$, there exists a path with four edges with the five ends being different. Three of these ends will be on one partition of the graph while the other two will be in the other partition. Take the vertex at the end of the path. It must be one of the three in the same partition. Since its degree is at least 2 , then it will have one neighbor not in the path. So, by adding the edge from the end of the path to this additional vertex, we obtain a new path with 5 edges with all its vertices being different. By taking the first, third, and fifth edges of this new path we obtain the result.

This lemma tells us that the vertices $\left\{x_{1}, \ldots, x_{v}\right\},\left\{y_{1}, \ldots, y_{b}\right\}$ in a $(v, b, r, k)$ configuration with $r \geq 3$ can be arranged in a way such that the edges $x_{1} y_{1}, x_{2} y_{2}$ and $x_{v} y_{b}$ belong to the configuration.

Suppose we have a $(v, b, r, k)$-configuration with vertices $\left\{x_{1}, \ldots, x_{v}\right\},\left\{y_{1}, \ldots, y_{b}\right\}$ and a $\left(v^{\prime}, b^{\prime}, r, k\right)$-configuration with vertices $\left\{x_{1}^{\prime}, \ldots, x_{v}^{\prime}\right\},\left\{y_{1}^{\prime}, \ldots, y_{b}^{\prime}\right\}$. Consider the graph with vertices $\left\{x_{1}, \ldots, x_{v}\right\} \cup\left\{x_{1}^{\prime}, \ldots, x_{v}^{\prime}\right\},\left\{y_{1}, \ldots, y_{b}\right\} \cup\left\{y_{1}^{\prime}, \ldots, y_{b}^{\prime}\right\}$ and all the edges in the original configurations. Swap the edges $x_{v} y_{b}$ and $x_{1}^{\prime} y_{1}^{\prime}$ for $x_{v} y_{1}^{\prime}$ and $x_{1}^{\prime} y_{b}$. This gives a $\left(v+v^{\prime}, b+b^{\prime}, r, k\right)$ configuration [2]. This construction proves the next lemma.

Lemma 5. If $(v, b, r, k),\left(v^{\prime}, b^{\prime}, r, k\right)$ are configurable tuples, so is $\left(v+v^{\prime}, b+b^{\prime}, r, k\right)$.

Lemma 6. $D_{r, k}$ satisfies

- $0 \in D_{r, k}$

- If $d, d^{\prime} \in D_{r, k}$ then $d+d^{\prime} \in D_{r, k}$.

Proof. Obviously $0 \in D_{r, k}$ and, by Lemma [5 if $d, d^{\prime} \in D_{r, k}$ then $d+d^{\prime} \in D_{r, k}$.

In order to have a numerical semigroup it remains to see that the number of elements in $\mathbb{N}_{0} \backslash D_{r, k}$ is finite. This will be proved in the next theorem. In the proof of the theorem it is used that two coprime integers generate a numerical 
semigroup and so, if a subset containing 0 and closed under addition contains two coprime integers then it is a numerical semigroup.

Theorem 2. $D_{r, k}$ is a numerical semigroup.

Proof. Because of the results in the previous sections we can assume that $r$ and $k$ are at least 3. By Lemma 3 and since $D_{r, k} \subseteq \mathbb{N}$, there is a minimal non-zero element $m$ in $D_{r, k}$. Let us call $v=m k / \operatorname{gcd}(r, k)$ and $b=m r / \operatorname{gcd}(r, k)$. Select a $(v, b, r, k)$ configuration. Take $s=r k / \operatorname{gcd}(r, k)$ copies of this configuration. Let us call the vertices of the $i$ th copy $x_{1}^{(i)}, \ldots, x_{v}^{(i)}, y_{1}^{(i)}, \ldots, y_{b}^{(i)}$. By Lemma 4 we can assume that $x_{1}^{(i)} y_{1}^{(i)}, x_{2}^{(i)} y_{2}^{(i)}$ and $x_{v}^{(i)} y_{b}^{(i)}$ belong to the $i$ th copy. Consider $k / \operatorname{gcd}(r, k)$ further vertices $x_{1}^{\prime}, \ldots, x_{k / \operatorname{gcd}(r, k)}^{\prime}$ and $r / \operatorname{gcd}(r, k)$ further vertices $y_{1}^{\prime}, \ldots, y_{r / \operatorname{gcd}(r, k)}^{\prime}$. For all $i<s$ swap the edges $x_{v}^{(i)} y_{b}^{(i)}$ and $x_{1}^{(i+1)} y_{1}^{(i+1)}$ for $x_{v}^{(i)} y_{1}^{(i+1)}$ and $x_{1}^{(i+1)} y_{b}^{(i)}$. Remove the edges $x_{2}^{(i)} y_{2}^{(i)}$ for all $i \leq s$. Add the edges

$$
\begin{gathered}
x_{1}^{\prime} y_{2}^{(1)}, x_{1}^{\prime} y_{2}^{(2)}, \ldots, x_{1}^{\prime} y_{2}^{(r)}, \\
x_{2}^{\prime} y_{2}^{(r+1)}, x_{2}^{\prime} y_{2}^{(r+2)}, \ldots, x_{2}^{\prime} y_{2}^{(2 r)}, \\
\vdots \\
x_{k / \operatorname{gcd}(r, k)}^{\prime} y_{2}^{(s-r+1)}, \ldots, x_{k / \operatorname{gcd}(r, k)}^{\prime} y_{2}^{(s)}
\end{gathered}
$$

and

$$
\begin{gathered}
x_{2}^{(1)} y_{1}^{\prime}, x_{2}^{(2)} y_{1}^{\prime}, \ldots, x_{2}^{(k)} y_{1}^{\prime}, \\
x_{2}^{(k+1)} y_{2}^{\prime}, x_{2}^{(k+2)} y_{2}^{\prime}, \ldots, x_{2}^{(2 k)} y_{2}^{\prime}, \\
\vdots \\
x_{2}^{(s-k+1)} y_{r / \operatorname{gcd}(r, k)}^{\prime}, \ldots, x_{2}^{(s)} y_{r / \operatorname{gcd}(r, k)}^{\prime} .
\end{gathered}
$$

It is easy to check that this is a new configuration with parameters

$$
\left(s v+\frac{k}{\operatorname{gcd}(r, k)}, s b+\frac{r}{\operatorname{gcd}(r, k)}, r, k\right)=\left(\frac{(s m+1) k}{\operatorname{gcd}(r, k)}, \frac{(s m+1) r}{\operatorname{gcd}(r, k)}, r, k\right)
$$

and so $s m+1 \in D_{r, k}$.

Since $m$ and $s m+1$ are coprime, they generate a numerical semigroup and this semigroup is contained in $D_{r, k}$. So the complement of $D_{r, k}$ in $\mathbb{N}_{0}$ is finite and $D_{r, k}$ is a numerical semigroup.

As a consequence of the fact that the necessary conditions P1, P2 are also sufficient for $k=3$ it is easy to deduce that $D_{r, k}=\{0\} \cup\left(\frac{2 r+1}{3} \operatorname{gcd}(3, r)+\mathbb{N}_{0}\right)$. The computation of examples for $r, k>3$ is computationally very hard.

\section{Conclusion}

The main conclusion of this short note is that for fixed $r$ and $k$ there exist configurations for all parameters $b, v$ large enough provided that $v r=b k$.

Another important fact is that our proofs are all constructive and so we can derive algorithms for constructing large configurations. 


\section{REFERENCES}

[1] F. De Clerck, J. A. Thas, and H. Van Maldeghem. Generalized polygons and semipartial geometries, 1996. EIDMA minicourse.

[2] J. Domingo-Ferrer, M. Bras-Amorós, Q. Wu, and J. Manjón. User-private information retrieval based on a peer-to-peer community. Data Knowl. Eng., 68(11):1237-1252, 2009.

[3] Harald Gropp. Non-symmetric configurations with deficiencies 1 and 2. Annals of Discrete Mathematics, 52:227-239, 1992.

[4] Harald Gropp. Nonsymmetric configurations with natural index. Discrete Math., 124(1-3):8798, 1994. Graphs and combinatorics (Qawra, 1990).

[5] Harald Gropp. Existence and enumeration of configurations. Bayreuth. Math. Schr., (74):123$129,2005$.

[6] Harald Gropp. Handbook Of Combinatorial Designs (Charles J. Colbourn and Jeffrey H. Dinitz ed.), chapter Configurations, pages 353-355. Chapman and Hall/CRC, Kenneth H. Rosen, 2007.

[7] Branko Grünbaum. Configurations of points and lines, volume 103 of Graduate Studies in Mathematics. American Mathematical Society, Providence, RI, 2009.

[8] J. C. Rosales and P. A. García-Sánchez. Numerical semigroups, volume 20 of Developments in Mathematics. Springer, New York, 2009.

[9] H. Sachs. Regular graphs with given girth and restricted circuits. J. London Math. Soc., 38:423-429, 1963. 